\title{
Variation in latency times of visually evoked cortical potentials
}

\author{
G. H. M. VAN LITH, G. W. VAN MARLE AND G. T. M. VAN DOK-MAK \\ From the Eye Hospital, Erasmus University, Rotterdam
}

SUMMARY Latency times of visually evoked cortical potentials stimulated by reversal of a slow checkerboard pattern are highly dependent on the time needed to accomplish the reversal movement. If, owing to the method, the pattern reversal time is not kept stable, variability of the latency times is unnecessarily high for clinical purposes. This may be the case when television equipment is used.

Halliday et al. $(1972,1973)$ showed that measurement of latency times of the visually evoked cortical potentials (VECPs), elicited by the reversal of a slow checkerboard pattern, is a very sensitive method of detecting disease in the visual pathways, especially in cases of optic neuritis and multiple sclerosis. Later Asselman et al. (1975) confirmed these findings. With this method of measuring latency times problems may occur, one of which is caused by the method used. We fell into this trap and by publishing our experience hope to help others avoid it.

Television systems are increasingly used to produce pattern stimuli, since they are more versatile in respect of size of check pattern, modulation depth, and mean luminance than other methods (Arden and Faulkner, 1977). Applying a TV system to determine latency times as Halliday did to diagnose multiple sclerosis, we found that the spreading of the normal range was much greater than his data showed. Consequently, we compared the latency times obtained by our TV system with those obtained by Halliday's methods.

In experiments with the TV system, the pattern had a check size of $20^{\prime}, 40^{\prime}$, or $80^{\prime}$, a modulation depth of $98 \%$, a mean luminance of $180 \mathrm{asb}$, and a field size of approximately $26^{\circ}$. Since no differences in the standard errors were found between the 3 check sizes used, only those obtained with the $40^{\prime}$ check size are mentioned here.

The apparatus as used by Halliday is a projector with a checkerboard slide and a moving mirror in front of it (Cobb et al., 1967). Its check size was $1^{\circ}$, the modulation depth $80 \%$, the mean luminance 400 or $40 \mathrm{asb}$, and the field size $28^{\circ}$. In both groups of experiments the reversal frequency was $2 \mathrm{~Hz}$ (periodicity $1 \mathrm{~Hz}$ ), the band width of the amplifiers

Address for reprints: Mr G. H. M. van Lith, Electroophthalmological Department, Eye Hospital, Schiedamsevest 180, 3000 LM Rotterdam, The Netherlands. was $0 \cdot 16$ to $75 \mathrm{~Hz}$, and 125 counts were averaged with an analysis time of $500 \mathrm{~ms}$. The electrical potentials were led off from surface electroencephalograph electrodes positioned at 5 and $15 \%$ above the inion in the midline referential to the earlobe, as well as bipolar from 5 to $25 \%$ (10 to $20 \%$ EEG - system). Twenty normal subjects were examined.

\section{Results}

An example of the recordings obtained with the 2 methods is shown in Fig. 1; amplitude and latency time were measured as indicated. In Table 1 the data of the 2 methods are compared. It appears that the amplitudes of the projector system are somewhat higher and the latency times somewhat longer than those of the TV system. These differences may be due to differences in check size and mean luminance. Much more impressive, however, is the large standard deviation (SD) in latency times obtained with the TV system, which is about twice as high as that of the projector system. Standard deviations of the amplitudes relative to the mean value are approximately the same.

A large standard deviation in latency time was also found when bipolar leads were applied instead of referential leads (Table 2). By decreasing the mean luminance the latency time itself is lengthened and the standard errors are not much influenced. On the amplitudes and their standard errors the mean luminance had not much influence either, both being larger in the referential leads than in the bipolar leads (Table 3).

\section{Discussion}

It is evident that the large differences in standard errors between the 2 methods cannot be explained 


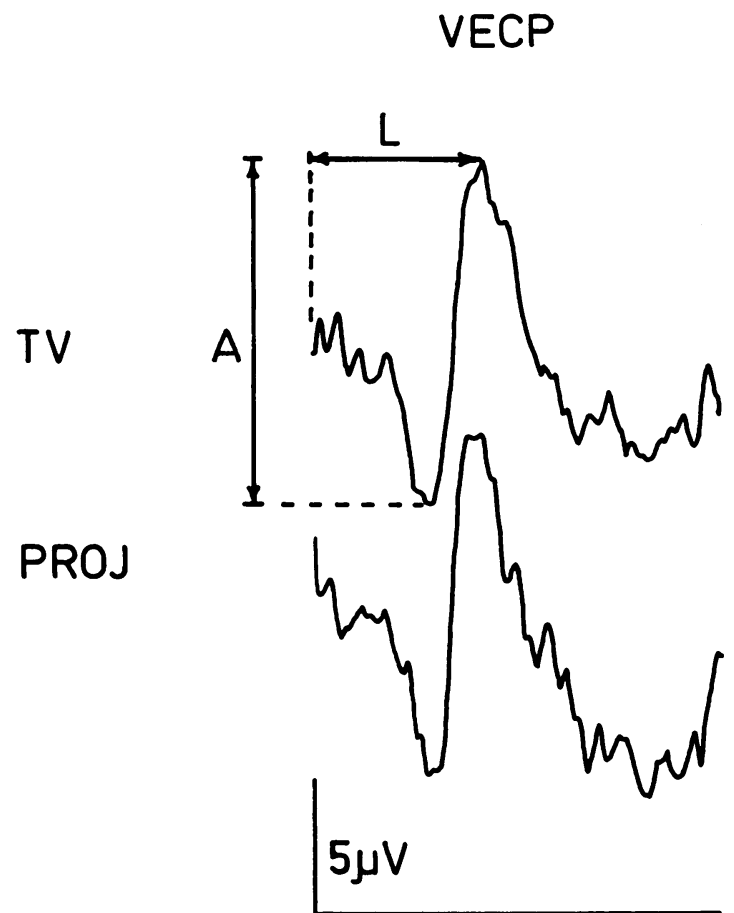

\section{$250 \mathrm{~ms}$}

Fig. 1 Visually evoked cortical potentials after a slow checkerboard pattern reversal stimulation of $1 \mathrm{~Hz}$, obtained with the TV system (upper curve) and with the projector system (lower curve)

by the relatively small differences in check size and modulation depth, nor by the differences in mean luminance or field size. When check size was varied, it did not seem to influence the standard error, nor did the mean luminance. The field size is relevant only inside a $20^{\circ}$ visual field, since visually evoked potentials stimulated by patterns are mediated by the central part of the retina only (Bartl et al., 1978).

The large standard error of readings from the bipolar leads is probably due to a summation effect of the standard errors of the 2 active electrode positions used, a summation of the values found in the referential leads.

As to the TV system, another and very logical explanation can be given. Asselman et al. (1975) reported that the latency time was influenced by the speed with which the reversal of the pattern was completed. In the projector system this time depends on the mirror movement in front of the projector, in the TV system on the frame frequency of the TV screen. In Asselman's set-up the mirror movement took $5 \mathrm{~ms}$, in our projector system $6 \mathrm{~ms}$, while it lasts $20 \mathrm{~ms}$ before a reversal movement is completed on the TV screen. Furthermore, in a projector system the whole pattern picture moves at the same time, whereas on the TV screen the pattern reversal is not a real movement but a replacement which starts in the left upper corner and is finished in the right lower corner $20 \mathrm{~ms}$ later, provided the starting point of the replacement is synchronised with the frame of the TV screen. Such synchronisation, however, causes interference with $50 \mathrm{~Hz}$ signals, which are then averaged too.

For this reason we chose to work with asynchronous signals-i.e., the pattern reversal starts independently from the frame, therefore starts somewhere at random on the screen. How long the measured latency time will be now depends on the time lapse between the starting point of the pattern reversal, which is also the trigger point of the averager, and the moment the reversal movement enters the 'evoked potential visual field' or 'trigger point' of the evoked potentials. By the 'EP visual field' is meant that part of the visual field, from which the pattern evoked potentials are mediated. This time lapse can be $20 \mathrm{~ms}$ maximum and $0 \mathrm{~ms}$ minimum. The averaged mean will be

Table 1 Latency times and amplitudes, obtained with a referential lead and in the projector method a mean luminance of 400 asb

\begin{tabular}{lll}
\hline & Projector & $T V$ \\
\hline Latency & $98.29 \mathrm{~ms}$ & $94.27 \mathrm{~ms}$ \\
& SE 8.63 & SE 16.04 \\
Amplitude & $9.11 \mu \mathrm{V}$ & $6.9 \mu \mathrm{V}$ \\
& SE 4.08 & SE 2.93 \\
\hline
\end{tabular}

Table 2 Latency times obtained with the projector method at 2 different luminances from bipolar and referential leads

\begin{tabular}{lll}
\hline & 400 asb & 40 asb \\
\hline Bipolar & $109.7 \mathrm{~ms}$ & $116 \mathrm{~ms}$ \\
& SE 19.45 & SE 20.76 \\
Referential & $98.29 \mathrm{~ms}$ & $110.93 \mathrm{~ms}$ \\
& SE 8.63 & SE 7.61 \\
\hline
\end{tabular}

Table 3 Amplitudes obtained with the projector method at 2 different luminances from bipolar and referential leads

\begin{tabular}{lll}
\hline & 400 asb & 40 asb \\
\hline Bipolar & $6.2 \mu \mathrm{V}$ & $5.62 \mu \mathrm{V}$ \\
& SE 2.85 & SE 2.65 \\
Referential & $9.11 \mu \mathrm{V}$ & $8.10 \mu \mathrm{V}$ \\
& SE 4.08 & SE 3.89 \\
\hline
\end{tabular}


somewhat less than $10 \mathrm{~ms}$, by which the difference in standard errors between the 2 systems is sufficiently explained.

The jitter in latency time, using a TV system can be improved, as mentioned above, by synchronising pattern reversal and frame, were it not that $50 \mathrm{~Hz}$ signals probably via the TV set seriously impaired the evoked potentials. Furthermore, if the fixation is not accurately and constantly in the middle of the screen, variations in latency times may also occur. This implies that TV systems like ours are less suitable for latency measurements than projector systems.

The influence of the mean luminance on the latency time itself needs attention if patients are examined with narrow or wide pupils. The relative difference of retinal illumination, being dependent on the pupil size $\left(\pi r^{2}\right)$, between a pupil of 2 and $8 \mathrm{~mm}$ amounts to more than $1 \log$ unit.

\section{References}

Arden, G. B., and Faulkner, D. J. (1977). A versatile pattern generator for neuro-ophthalmological and paediatric EP and psychophysical tests using standard television techniques compatible with broadcast colour programmes. In Experimental and clinical amblyopia; Proceedings XIIIth ISCERG Symposium, Israel, 1975. Documenta Ophthalmologica Proceedings Series, Vol. 11, pp. 165-166. Edited by E. Auerbach.

Asselman, P., Chadwick, D. W., and Marsden, C. D. (1975). Visual evoked responses in the diagnosis and management of patients suspected of multiple sclerosis. Brain, 98, 261-282.

Bartl, G., et al. (1978). To be published.

Cobb, W. A., Morton, H. B., and Ettlinger, G. (1967). Cerebral potentials evoked by pattern reversal and their suppression in visual rivalry. Nature, 216, 1123-1125.

Halliday, A. M., McDonald, W. I., and Mushin, J. (1972). Delayed visual evoked response in optic neuritis. Lancet, 1, 982-985.

Halliday, A. M., McDonald, W. I., and Mushin, J. (1973). Visual evoked response in diagnosis of multiple sclerosis. British Medical Journal, 4, 661-664. 